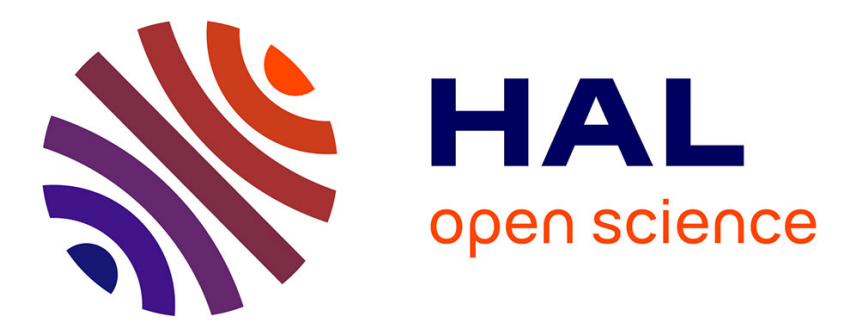

\title{
Cancer des VADS et comportements à risque
}

Guillaume Grandazzi, Emmanuel Babin, Marie van Der Schueren

\section{To cite this version:}

Guillaume Grandazzi, Emmanuel Babin, Marie van Der Schueren. Cancer des VADS et comportements à risque. Psycho-Oncologie, 2017, 11 (4), pp.233-237. 10.3166/s11839-017-0634-x . hal-02130365

\section{HAL Id: hal-02130365 \\ https://hal-normandie-univ.archives-ouvertes.fr/hal-02130365}

Submitted on 21 May 2019

HAL is a multi-disciplinary open access archive for the deposit and dissemination of scientific research documents, whether they are published or not. The documents may come from teaching and research institutions in France or abroad, or from public or private research centers.
L'archive ouverte pluridisciplinaire HAL, est destinée au dépôt et à la diffusion de documents scientifiques de niveau recherche, publiés ou non, émanant des établissements d'enseignement et de recherche français ou étrangers, des laboratoires publics ou privés. 


\title{
Cancer des VADS et comportements à risque
}

\author{
Head and Neck Cancers and Risk Behaviors
}

E. Babin · G. Grandazzi • M. Van der Schueren

Résumé Les cancers des voies aérodigestives supérieures (VADS) sont majoritairement des cancers masculins. Ils sont associés avant tout à une consommation excessive d'alcool et de tabac. Ils apparaissent comme des sociopathologies. Les répercussions psychosociales de ces cancers se manifestent par des trajectoires de vie difficiles et une dégradation des relations au sein du couple. Ces constatations incitent à mettre en œuvre des politiques de santé contre le tabagisme et à proposer des recherches pour réduire l'incidence de ces cancers.

Mots clés Cancers des voies aérodigestives supérieures · Cancers masculins · Tabac et alcool · Sociopathologie

\begin{abstract}
Head and neck cancers are predominantly male cancers. They are associated with excessive alcohol and tobacco consumption. They can be considered as sociopathologies. The psychosocial consequences of these cancers are linked with difficult life trajectories and a deterioration of relationships within the couple. These findings encourage the implementation of anti-smoking health policies and the provision of research to reduce the incidence of these cancers.
\end{abstract}

Keywords Head and neck cancers $\cdot$ Male cancers $\cdot$ Tobacco and alcohol $\cdot$ Sociopathology

\section{Introduction}

Les cancers des voies aérodigestives supérieures (VADS), si on exclut les cancers du sinus et du cavum, sont majoritaire ment des cancers masculins. En 2012, l'incidence mondiale de ces cancers était chez les hommes de 21,5 cas pour 100000 personnes par an [1]. Ces cancers de la tête et du cou représentent en Europe le quatrième cancer masculin [2]. En France, l'incidence de ces cancers était de 16000 nouveaux cas par an et constituait la cinquième cause de décès chez l'homme [3]. Le nord-ouest de la France affiche un taux élevé avec 35 cas pour 100000 personnes par an [4]. Ce taux d'incidence a toutefois diminué en France depuis $1980 \mathrm{chez} \mathrm{l'homme,} \mathrm{mais} \mathrm{a}$ doublé chez la femme pendant la même période [3].

Ces cancers sont diagnostiqués à des stades avancés (stades III-IV) dans plus des deux tiers des cas et associés à des taux de survies à cinq ans ( $28 \%$ pour les cancers de l'hypopharynx et $57 \%$ pour les cancers du larynx) [5]. Ils surviennent généralement autour de 50-60 ans dans des populations à faible revenu économique. L'environnement et les habitudes de vie des individus avec la consommation de tabac et d'alcool sont étroitement liés à l'apparition de ce type de cancer. L'analyse du quotidien et du milieu de vie de ces patients peut expliquer la prédisposition à la consommation de toxiques générateurs de cancers.

L'objectif de cet article est de définir les comportements à risque liés aux cancers des VADS. L'identification de ces éléments replacés dans l'environnement social peut permettre de cibler des actions prioritaires et d'élaborer une stratégie de prise en charge de ces patients afin de réduire les conséquences de ces traitements sur le quotidien des patients. 


\section{Facteurs de risques environnementaux}

Le tabagisme chronique serait responsable de 54 à $87 \%$ des cancers des VADS [6,7]. L'alcool constitue le deuxième facteur de risque. Périé et al. estiment à $168 \%$ l'augmentation du risque de cancer de la cavité buccale, du larynx et du pharynx par verre de boisson alcoolisée consommé au quotidien [3]. La consommation de ces deux toxiques multiplie les risques de développer un cancer. Dans le cadre des cancers de la cavité buccale, il est de 1,66 pour un consommateur régulier d'alcool, de 1,57 pour un fumeur qui ne consomme pas d'alcool, et de 13 en présence d'une consommation combinée [8].

En marge de cette double intoxication existe la consommation de stupéfiants notamment la marijuana qui est plutôt associée à la survenue de cancers de la cavité buccale (langue) [9]. Ces lésions apparaissent chez des patients plus jeunes et dépendent de la dose et de la durée d'exposition. La consommation d'opium serait un facteur de risque pour le larynx. Il est toutefois difficile d'isoler ces stupéfiants comme facteur de risque indépendant. La majorité des patients multiplie les expositions au tabac, alcool et autres stupéfiants [10].

L'infection par HPV constitue un autre facteur de risque reconnu de survenue de ce type de cancer. Le rôle des virus Human Papilloma Virus (HPV) dans la carcinogenèse est bien identifié pour les localisations cancéreuses oropharyngées (30 à $50 \%$ au sein des amygdales) [7]. Ces cancers sont davantage retrouvés chez les hommes dont la compagne est atteinte d'un cancer du col utérin. Ils affichent quelques particularités cliniques : les patients sont plus jeunes, consomment moins de tabac, les partenaires et pratiques sexuelles sont multiples (notamment orogénitales).

D'autres causes non liées à l'environnement comportemental peuvent être incriminées. Certains facteurs génétiques sont cités de même que des facteurs nutritionnels (déficit en fer) et/ou des carences vitaminiques en vitamines $\mathrm{A}$ et $\mathrm{C}$ ainsi que le reflux gastro-œsophagien, l'immunodépression parle virus VIH [11]. Des expositions professionnelles (nickel, polyvinyles, vapeurs de diesel, aérosols d'huile, amiante) sont avancées, mais difficiles à mettre en évidence compte tenu de la fréquence d'exposition croisée avec l'alcool et le tabac.

\section{Cancer des VADS comme sociopathologie}

Le tabac et l'alcool sont les principaux inducteurs de cancers des VADS. Ils traduisent aussi le poids des inégalités sociales devant la maladie cancéreuse. Desplanques [12] a démontré la plus grande exposition des ouvriers à développer un cancer en relation avec une intoxication alcoolotabagique. Dans les années 1980, le taux de mortalité par tumeur du larynx pour les hommes de 45 à 54 ans variait de 1 à 11 entre les professeurs et l'ensemble des ouvriers spécialisés et des manœuvres [12]. Cette différence perdurait 20 ans plus tard [13]. La consommation de ces toxiques en vente libre peut être assimilée à la manifestation d'un malaise social. L'épuisement physique et psychologique lié aux conditions de vie, à « la fatigue d'être soi » [14], le mal-être au travail sont autant de manifestations de la façon dont « la santé et la maladie reflètent nos 
interactions avec l'environnement » [15].

Les processus observés avec la maladie néoplasique sont notamment déclenchés par des pensées, des sentiments et des comportements associés à la situation matérielle et à la position sociale. Ces situations d'individus épuisés moralement par leur vie expliquent en partie les raisons d'une intoxication éthylotabagique chronique [16], qui constitue un moyen facile et accessible pour accepter les difficultés liées à des biographies parfois chaotiques et s'évader d'une existence imparfaite. L'impact de l'environnement social, économique et professionnel sur la consommation d'alcool et de tabac, facteurs de risque importants pour les cancers des VADS, permet de considérer ces derniers comme des sociopathologies.

Pour autant, les patients atteints de ce type de cancers n'en subissent pas moins une stigmatisation qui se concrétise sous deux formes majeures et complémentaires : rejet social et condamnation morale. En effet, comme cela a été montré par d'autres chercheurs [17], les patients atteints de cancers associés à la consommation de tabac et d'alcool, comme le cancer du poumon ou des VADS, se voient davantage stigmatisés par les soignants et leurs proches, quand bien même ils ne consomment pas ces substances. Fumer et boire de l'alcool s'apparentent, dans la plupart des pays occidentaux, à des comportements jugés comme déviants et moralement condamnables, ce qui retentit sur la façon dont sont stigmatisés ensuite les malades, qui doivent en outre faire face pour une partie d'entre eux à la stigmatisation liée aux conséquences du cancer et des traitements qui détériorent leur image corporelle, et en particulier leur visage pour les cancers des VADS.

Une étude réalisée dans le nord-ouest de la France auprès de 270 conjoints de patients atteints d'un cancer des VADS et interrogés par questionnaires dans l'année qui a suivi le diagnostic a montré que plus de $40 \%$ d'entre eux considèrent que les habitudes de vie, et notamment la consommation d'alcool et de tabac, ont favorisé l'apparition du cancer de leur proche. Pourtant, l'annonce du diagnostic de cancer a constitué une surprise pour plus de trois quarts d'entre eux.

Une approche qualitative complémentaire par entretiens semi-directifs auprès des conjoints de patients a permis quant à elle de mieux comprendre les conséquences de l'éthylotabagisme sur la vie conjugale, avant et avec ou après la maladie.

Souvent liée à des trajectoires de vie difficiles, la consommation de ces produits à des doses importantes et dans la longue durée conduit le plus souvent à une dégradation des relations au sein du couple, la survenue de la maladie s'inscrivant alors dans un contexte de tensions entre les conjoints, exacerbées par la culpabilité et le ressentiment, comme en témoignent certains propos recueillis auprès des proches :

"Le problème ce n'est même pas le cancer, c'est ça qui me dérange, il s'est foutu en l'air, il s'est foutu en l'air avec la cigarette "; " il l'a cherché lui, parce qu'il a bu, il a fumé, et ce n'est pas faute de lui avoir dit »;

" je lui dis : "toi, ce que tu as là au jour d'aujourd'hui, eh bien si tu n'avais pas fumé peut-être que tu ne l'aurais pas" "; "ça détruit hein... On s'en veut... I" m'a reproché d'être malade... II me culpabilisait, donc moi je lui ai dit : "non je suis désolée, ce n'est pas moi qui fumais, ce n'est pas moi qui buvais donc ce n'est pas ma faute, c'est la tienne. Et en plus, tu nous as 
enfumés aussi, mes enfants et moi". Donc c'est vrai qu'on se dit des choses méchantes, quand on est un peu colère comme ça ".

Les conjoints jouent un rôle important dans la gestion des trajectoires de maladie en favorisant l'abstinence du malade lorsqu'il a arrêté sa consommation après le diagnostic ou pendant les traitements, pour éviter toute reprise de l'intoxication associée à l'idée de récidive. Posée parfois comme une condition à la continuité du travail de soin effectué par le proche, voire à la poursuite de la relation conjugale, l'abstinence constitue un enjeu important dans la vie avec le cancer, d'autant plus quand il s'agit d'un combat entamé bien avant la maladie, comme pour cette femme qui s'était battue avec succès par le passé contre l'alcoolisme de son mari :

"Une fois qu'il était mieux, il a eu des petites envies, je lui ai dit : "si tu fumes, tu fais ce que tu veux, tu sais ce qui te pend au bout du nez, mais moi cette fois-ci, je te préviens, tu fumes, tu rechutes, tu te débrouilles, moi je ne m'occupe plus de toi. Il peut arriver quoi que ce soit, moi je ne vais même plus te voir à l'hôpital, je ne fais plus rien". "

Une autre femme interrogée, dont le mari s'est remis à boire deux ans après le diagnostic, fait part de son incompréhension et de sa déception, mais également de son impuissance liée à la culpabilité éprouvée à l'idée de quitter son mari ou de ne plus s'en occuper :

" Je ne comprends pas, au bout de deux ans, se remettre à boire comme ça... et les deux ans où il ne buvait plus une goutte d'alcool ce n'était vraiment pas le même. La vie, c'était tout pour lui, mais là maintenant, il se laisse aller... Je serais même à la limite de m'en aller, avec ma fille. Mais je ne le fais pas parce que je ne veux pas le laisser tout seul. Ce serait encore pire, je crois. "

Les médecins, généralistes ou spécialistes, qui prennent en charge ces patients peuvent également jouer un rôle essentiel en faveur de la réduction ou de l'arrêt de la consommation d'alcool et de tabac en tenant à leurs patients des discours clairs et sans ambages, comme le raconte un patient abstinent depuis cinq ans, peut-être grâce aux propos directs que lui a tenus son praticien :

" II m'a dit : "si tu te remets à boire et à fumer, ce n'est pas la peine de revenir me voir. J'irai te revoir, mais pas à la polyclinique, j'irai te revoir au cimetière". Et je n'ai jamais refumé ni retouché à l'alcool. »

\section{Perspectives}

Les chapitres précédents ont montré les liens qui existent entre les cancers des VADS, le sexe masculin, l'intoxication alcoolotabagique et certains facteurs environnementaux et/ ou comportements individuels.

Dans l'exemple du tabac, arrêter de fumer est aussi indispensable lors de la survenue d'un cancer d'autant plus que si la personne est guérie de son premier cancer et continue à fumer, elle a un très grand risque de faire une seconde localisation (risque multiplié par 22 d'un second cancer après un premier cancer du sein traité par radiothérapie sans arrêt du tabac). 
Une méta-analyse [18] a montré chez une personne qui continue à fumer après le diagnostic de cancer un risque multiplié par trois de mortalité toutes causes confondues. Si elle ne meurt pas tout de suite, elle va doubler ses risques de reprises évolutives de la maladie. La poursuite du tabac a aussi des effets secondaires sur les traitements du cancer avec une augmentation du risque de complications per- et postopératoires et accroit les effets secondaires de la radiothérapie [19]. Elle diminue également la qualité de vie, les fumeurs persistant pendant les traitements présentent plus de nausées, de vomissements, de dyspnée, de diarrhées, ont moins d'appétit et perdent plus de poids [20].

L'arrêt du tabac est souhaité chez $68 \%$ des fumeurs dans les six mois après la découverte de leur cancer, alors que le baromètre santé de la population générale retrouve des intentions beaucoup plus faibles [21]. Avec le patient, la famille et l'entourage proche doivent eux aussi être impliqués. Le moment idéal pour capter l'attention des acteurs est l'annonce d'un diagnostic est ce qu'on appelle un teachable moment, c'est-à-dire un «moment clé » pour parler du sevrage.

Il faut remettre le patient et son entourage au cœur de la démarche et la notion d'apprentissage d'un nouveau comportement est intéressante en termes de stratégie d'approche. Permettre aux patients d'acquérir de nouvelles compétences : c'est l'éducation thérapeutique du patient (ETP).

Le programme sera en trois points :

- le savoir agir qui suppose de savoir combiner et mobiliser des ressources pertinentes (connaissances, savoir-faire, réseaux) ;

- le vouloir agir qui se réfère à la motivation personnelle de l'individu et au contexte plus ou moins incitatif dans lequel il intervient ;

- le pouvoir agir qui renvoie à l'existence d'un contexte, d'une organisation du travail, de choix de management, de conditions sociales qui rendent possibles et légitimes la prise de responsabilité et la prise de risque de l'individu [22].

Une autre forme de lutte contre les conséquences des cancers des VADS passe par le dépistage et/ou une découverte précoce réduisant ainsi les prises en charge «lourdes».

Les campagnes de prévention sur les méfaits de l'alcool et du tabac sont nombreuses, majeures, récurrentes, mais n'ont pas apporté pour le moment de résultat probant sur une diminution de ces cancers.

Dans cette localisation cancéreuse, un des problèmes liés au dépistage réside dans l'intéressement du sujet « cible ». Fagnani avait démontré dans ses travaux que les personnes qui se rendaient au dépistage n'étaient pas celles exposées au risque le plus élevé ; en majorité, c'étaient les sujets qui ne venaient pas régulièrement consulter leur médecin qui présentaient les risques les plus élevés [23]. Quarante ans plus tard, rien n’a changé. Les populations de tabagiques chroniques et de consommateurs d'alcool se prêtent peu aux examens «informatifs ». Des campagnes annuelles existent type makesenscampaign.eu, mais affichent des résultats décevants. Les experts médicaux recrutent davantage de patients cancérophobes que de malades de cancer de la «gorge ».

Les efforts d'intensification des diagnostics précoces laissent dubitatifs [24]. Le stade actuel de diagnostic pour ces cancers reste problématique : $65 \%$ des patients arrivent à des stades « avancés » classés III-IV, il faut donc tout mettre en œuvre pour rechercher ces cancers plus tôt. 
L'amélioration du diagnostic nécessite de sensibiliser les acteurs médicaux aux populations exposées. Les médecins généralistes sont en première ligne. Quatre-vingt-six pour cent des patients atteints d'un cancer des VADS ont consulté leur médecin généraliste au moins une fois un an avant le diagnostic de cancer [25]. Les Anglais ont mis en place une structure de diagnostic précoce. Langton et al. [26] montrent un taux de diagnostic positif dans des populations symptomatiques égal à 8,8\% illustré par la découverte de 360 cancers pour 4086 visites. Ce taux peut paraître bas, mais peut être amélioré en sensibilisant les acteurs lors de formations présentielles ou en e-learning. Hassona et al. [27] montrent que les facteurs associés à une amélioration du dépistage sont des médecins jeunes diplômés depuis moins de cinq ans et la participation à des formations continues.

La clé du diagnostic précoce à défaut de proposer un dépistage efficace est là. La responsabilisation des acteurs médicaux et en première ligne les médecins généralistes, leur implication dans l'encouragement à stopper les intoxications constituent des démarches à développer. Dans le territoire normand, une étude est en préparation avec l'Union des médecins régionaux libéraux (UMRL) pour instaurer un dispositif similaire à celui de Langton et al.

L'étape ultime résidera dans la mise au point de technique de dépistage simple adaptée pour ces cancers. L'analyse des composés volatils organiques est une piste en cours [28]. Toutes ces propositions ont un coût pour la société. Les pouvoirs publics doivent-ils continuer à les encourager ? Aucune étude à ce jour n'a montré l'amélioration de la survie avec un dépistage précoce des cancers des VADS. Cela justifie-t-il pour autant de ne rien faire ?

Enfin, dans le registre sociétal, les liens de cause à effet entre la survenue des cancers des VADS et les conditions de vie sont certes d'ordre comportemental, mais aussi d'ordre psychosocial, ce que ne prennent pas toujours en compte les spécialistes du corps médical. Ces derniers se sont souvent limités jusqu'à maintenant à identifier et à lutter contre les facteurs biologiques. Par conséquent, et à défaut de traiter la véritable étiologie du cancer, nos mesures de prévention restent souvent vaines, la dimension psychosociale étant éludée. Cette constatation reste une voie de recherche à développer.

\section{Conclusion}

Les cancers des VADS apparaissent clairement comme une sociopathologie. Les «principaux » fauteurs de troubles sont clairement le tabac et l'alcool en lien avec des comportements individuels catalysés par notre société.

Le combat contre cette maladie est un challenge à relever avec tous. Guérir médicalement du cancer est un objectif non encore atteint, mais qui le sera peut-être avec la révolution génomique. Dans ces conditions, les propositions de limiter les cancers passent par d'autres voies.

Lutter contre les lobbys des « cigarettiers » et des producteurs d'alcool reste voué à l'échec. Changer la société a été pensé par beaucoup, mais n'est qu'illusion. En revanche, nous pouvons sans doute agir pour développer la prévention secondaire, améliorer le diagnostic précoce de ces cancers et améliorer la qualité de vie des malades des cancers de la tête et du cou. 


\section{Références}

1. Ligier K, Belot A, Launoy G, et al (2011) Descriptive epidemiology of upper aerodigestive tract cancers in France: incidence over 1980-2005 and projection to 2010. Oral Oncol 47:3027

2. Ferlay J, Steliarova-Foucher E, Lortet-Tieulent J, et al (2013) Cancer incidence and mortality patterns in Europe: estimates for 40 countries in 2012. Eur J Cancer 49:1374-403

3. Périé S, Meyers M, Mazzaschi O, et al (2014) Épidémiologie et anatomie des cancers ORL. Bull Cancer 101:404-10

4. Ligier K, Plouvier S, Danzon A, et al (2012) Elements of completeness and results of the first year of registration of the "Registre général des cancers de Lille et de sa région". Rev Epidemiol Sante Publique 60:131-9

5. Guizard AV, Dejardin O, Launay L, et al (2017) Diagnosis and management of head and neck cancers in a high-incidence area in France: A population-based study. Medicine 96:e7285

6. Noms des auteurs à ajouter]] (2003) Rapport de Commission d'orientation sur le cancer. www.sante.gouv.fr/IMG/pdf/rapport_- commission_orientation_cancer.pdf

7. Sturgis EM, Cinciripini PM (2007) Trends in head and neck cancer incidence in relation to smoking prevalence: an emerging epidemic of human papillomavirus-associated cancers? Cancer 110:1429-35

8. Castellsagué X, Quintana MJ, Martinez MC, et al (2004) The role of type of tobacco and type of alcoholic beverage in oral carcinogenesis. Int J Cancer 108:741-9

9. Carriot F, Sasco AJ (2000) Cannabis and cancer. Rev Epidemiol Sante Publique 48:473-83

10. Verges S, Morinière S, Dubrulle F, et al (2013) Initial staging of squamous cell carcinoma of the oral cavity, larynx and pharynx (excluding nasopharynx). Part I ; locoregional extension assessment : 2012 SFORL guidelines. Eyr Ann Otolaryngol Head Neck Did 130: 39-45

11. Lewden C, May T, Rosenthal E, et al (2006) Causes de décès en France en 2005 des adultes infectés par le VIH et évolution par rapport à 2000. BEH 48:379-82

12. Desplanques G (1984) L'inégalité sociale devant la mort. Econ Stat 162:29-50

13. Babin E (2011) Le cancer de la gorge et la laryngectomie : la découration. L'Harmattan, Paris

14. Ehrenberg A (1991) Le culte de la performance. Calmann-Lévy, Paris 
15. Wilkinson R (2002) L'inégalité nuit gravement à la santé. Cassini, Paris

16. Fassin D (1996) L'espace politique de la santé. Essai de généalogie. Puf, Paris

17. Lebel S, Castonguay M, Mackness G, et al (2013) The psycho-social impact of stigma in people with head and neck or lung cancer. Psycho-Oncology 22:140-52

18. Parsons A, Daley A, Begh R, Aveyard P (2010) Influence of smoking cessation after diagnosis of early stage lung cancer on prognosis: systematic review of observational studies with meta- analysis. BMJ 340:b5569

19. Gaillot-de-Saintignon J, Deutsch A (2016) Systématiser l'accompagnement à l'arrêt du tabac pour améliorer le traitement des patients atteints de cancer. Bull Cancer 103:584-93

20. Jensen K, Jensen AB, Grau C (2007) Smoking has a negative impact upon health related quality of life after treatment for head and neck cancer. Oral Oncol 43:187-92

21. Guignard R, Beck F, Richard JB, et al (2015) La consommation de tabac en France en 2014 : caractéristiques et évolutions récentes, Évolutions 31:6 p

22. Le Boterf G (2006) Construire les compétences individuelles et collectives. Éditions d'Organisation, Paris

23. Fagnani S (1973) Santé, consommation médicale et environnement. Problèmes et méthodes, Mouton, Paris

24. Brocklehurst P, Kujan O, O’Malley LA, et al (2013) Screening programmes for the early detection and prevention of oral cancer. Cochrane Database Syst Rev 11:CD004150

25. Ligier K, Dejardin O, Launay L, et al (2016) Health professionals and the early detection of head and neck cancers: a population- based study in a high incidence area. BMC Cancer $16: 456$

26. Langton S, Siau D, Bankhead C (2016) Two-week rule in head neck cancer 2000-14: a systematic review. Br J Oral Maxillofac Surg 54:120-31

27. Hassona Y, Scully C, Shahin A (2016) Factors influencing early detection of oral cancer by primary health-care professionals. J Canc Educ 31:285-91

28. Gruber M, Tisch U, Jeries R, et al (2014) Analysis of exhaled breath for diagnosing head and neck squamous cell carcinoma: a feasibility study. Br J Cancer 111:790-8 